\title{
Hydrocortisone Dose Influences Pain, Depressive Symptoms and Perceived Health in Adrenal Insufficiency: A Randomized Controlled Trial
}

\author{
Jorien Werumeus Buning ${ }^{a}$ Pauline Brummelman ${ }^{a} \quad J^{2}$ nneke Koerts ${ }^{b}$ \\ Robin P.F. Dullaart ${ }^{a} \quad$ Gerrit van den Berg $^{a} \quad$ Melanie M. van der Klauw ${ }^{a}$ \\ Wim J. Sluiter ${ }^{a}$ Oliver Tuchab $^{b} \quad$ Bruce H.R. Wolffenbuttel ${ }^{\mathrm{a}}$ André P. van Beek $^{\mathrm{a}}$ \\ ${ }^{a}$ Department of Endocrinology, University of Groningen, University Medical Center Groningen, and ${ }^{b}$ Department of \\ Clinical and Developmental Neuropsychology, University of Groningen, Groningen, The Netherlands
}

\section{Key Words}

Hydrocortisone · Adrenal insufficiency · Quality of life · Pain · Depression · Fatigue

\begin{abstract}
Background: There is a major lack of randomized controlled trials (RCTs) evaluating the effects of hydrocortisone (HC) substitution therapy in patients with secondary adrenal insufficiency. Therefore, we evaluated the effects of two different replacement doses of $\mathrm{HC}$ on health-related quality of life (HRQoL) in a RCT. Methods: This RCT with a double-blind cross-over design was performed at the University Medical Center Groningen. Forty-seven patients ( 29 men, age $51 \pm 14$ years, range 19-73 years) with secondary adrenal insufficiency participated. Patients received both a lower and a higher dose of $\mathrm{HC}(0.2-0.3$ and $0.4-0.6 \mathrm{mg} / \mathrm{kg}$ body weight/day) for 10 weeks in random order. HRQoL was assessed with a daily mood and symptom checklist (Patient Health Questionnaire-15 [PHQ-15], Generalized Anxiety Disorder-7 [GAD-7], Patient Health Questionnaire-9 [PHQ-9]) and with questionnaires assessing general well-being (RAND 36-Item Health Survey [RAND-36]), mood (Hospital Anxiety and Depression Scale [HADS]) and fatigue (Multidimensional Fatigue Inventory-20 [MFI-20]). ClinicalTrials.gov identifier: NCT01546922. Results: Patients receiving the higher dose of $\mathrm{HC}$ reported
\end{abstract}

\section{KARGER}

E-Mail karger@karger.com

www.karger.com/nen significantly fewer symptoms of depression $(p=0.016$ and $p=0.045$ for HADS and PHQ-9, respectively), less general and mental fatigue $(p=0.004$ and $p=0.003$, respectively, both MFI-20), increased motivation ( $p=0.021, \mathrm{MFI}-20)$, better physical functioning $(p=0.041)$, better general health $(p=$ 0.013 ) and more vitality ( $p=0.025)$ (all RAND-36). In addition, while on the higher dose, fewer somatic symptoms ( $p=$ $0.022)$ and less pain $(p<0.001)$ (both PHQ-15) were experienced. Conclusions: On the higher dose of HC, patients reported a better HRQoL on various domains as compared to the lower dose of $\mathrm{HC}$. The fact that a higher dose of $\mathrm{HC}$ may improve patient well-being should be taken into consideration when individualizing the $\mathrm{HC}$ substitution dose.

(c) 2015 S. Karger AG, Basel

\section{Introduction}

Adrenal insufficiency (AI) requires lifelong daily medical treatment with glucocorticoids (GCs). The standard replacement therapy consists of the oral administration of GCs, usually hydrocortisone (HC), with the aim of mimicking the daily rhythm in cortisol concentrations seen in healthy people. However, there are no criteria to objectively monitor and evaluate the quality of substitution therapy $[1,2]$. Consequently, current practice varies
C 2015 S. Karger AG, Basel

0028-3835/15/1036-0771\$39.50/0
J. Werumeus Buning

University Medical Center Groningen

De Brug, AA31, P.O. Box 30.001

NL-9700 RB Groningen (The Netherlands)

E-Mail j.werumeus.buning@ umcg.nl 
widely with regard to the administered dose of GCs. This was recently highlighted in a large study reporting that $20 \%$ of patients received low daily doses $(<20 \mathrm{mg} \mathrm{HC}$ equivalent dose), $40 \%$ received intermediate doses and $40 \%$ received high doses ( $\geq 30 \mathrm{mg} \mathrm{HC}$ equivalent dose) [3].

Current HC dosing schemes are the result of a complex balancing of factors including the endogenous cortisol production as documented in healthy individuals, variation in plasma cortisol in relation to the $\mathrm{HC}$ substitution dose, and the risks and benefits of applying (longterm) higher or lower dosing schemes [2, 4-6]. Healthrelated quality of life (HRQoL) is another important issue in the individualization of the dosing scheme. HRQoL refers to subjective and multidimensional domains encompassing, for instance, physical functioning, psychological state and social interaction. As such, it is often a subject of discussion between physician and patient.

Cross-sectional studies suggest that there is a relationship between the mean daily dose of GCs and HRQoL, with higher GC doses being significantly related to more severely impaired subjective health status [7-10]. However, in view of the cross-sectional nature of these studies, it is impossible to distinguish whether diminished QoL is a result of a higher dose or, conversely, whether diminished QoL results in the prescription of a higher dose. A few controlled studies have assessed HRQoL in relation to HC dose. All studies were small and applied different $\mathrm{HC}$ regimens, often with changes to both timing and dosing [6]. These studies produced variable results, with increases [11], no change $[12,13]$ or decreases [14] in QoL being reported with higher doses of GCs. Thus the exact relation between $\mathrm{HC}$ dose and HRQoL remains unknown.

This randomized double-blind cross-over study was initiated to determine the effect of the total daily dose of $\mathrm{HC}$ on several indices of HRQoL by comparing a lower replacement dose of $\mathrm{HC}$ to a higher replacement dose of $\mathrm{HC}$ in patients with secondary AI.

\section{Patients and Methods}

\section{Patients}

For this randomized double-blind cross-over study patients were recruited from the endocrine outpatient clinic of the University Medical Center Groningen. A total of 63 patients were included, of whom 60 completed the run-in phase and the baseline measurement. Eligibility, inclusion and follow-up are shown in online supplementary figure 1 (for all online suppl. material, see www. karger.com/doi/10.1159/000442985). All patients had secondary $\mathrm{AI}$ and were on stable GC substitution therapy at least 6 months prior to study entry. The diagnosis of secondary AI was based on internationally accepted biochemical criteria, principally early morning (08.00-09.00 h) serum cortisol measurements and, if necessary, an insulin tolerance test. Early morning cut-off cortisol levels for AI in our center were validated for patients with hypothalamic-pituitary disorders as published previously [15]. Other pituitary hormone deficiencies were adequately replaced when necessary for at least 6 months prior to study entry and treatment remained stable during the study. Other inclusion and exclusion criteria have been described earlier [16]. All patients were tested in the period between May 2012 and June 2013.

\section{Intervention}

Group 1 first received a lower dose of $0.2-0.3 \mathrm{mg} \mathrm{HC} / \mathrm{kg}$ body weight/day (i.e. a total daily dose of 15-20 mg HC/day) divided into three doses (before breakfast, before lunch, before dinner) for 10 weeks, followed by a higher dose of $0.4-0.6 \mathrm{mg} \mathrm{HC} / \mathrm{kg}$ body weight/day (i.e. a total daily dose of 30-40 $\mathrm{mg} \mathrm{HC/day)} \mathrm{for} \mathrm{a} \mathrm{fur-}$ ther 10 weeks. Group 2 received the two doses in reversed order. For the exact dosing scheme see online supplementary table 1 . Randomization to one of the two treatment groups was performed by Tiofarma Inc., The Netherlands, with a block size of four. In cases of intercurrent illness or fever, patients were advised to double or triple their $\mathrm{HC}$ dose according to a fixed protocol. In the last week before testing this was not allowed.

After each treatment period patients returned to the hospital to hand in questionnaires and diaries. In addition, blood samples were drawn, both $1 \mathrm{~h}$ and $5 \mathrm{~h}$ after ingestion of the morning dose of HC.

\section{Withdrawals}

A total of 63 patients were included in the study. During the run-in phase, 3 patients withdrew from the study; none of these withdrawals was suspected to be related to the dose of $\mathrm{HC}$ ( 2 patients withdrew their informed consent and 1 patient was withdrawn by the investigator because of the presence of chronic pain syndrome). Eight patients withdrew from the study during the lower dose condition; 3 of those withdrawals were suspected to be related to the dose of $\mathrm{HC}$ (influenza A infection [ $\mathrm{n}=1$ ], inability to tolerate the dose $[n=1]$ and Herpes zoster ophthalmicus infection $[n=1])$. Five patients withdrew from the study during the higher-dose condition; one of these withdrawals was suspected to be related to the dose of $\mathrm{HC}$ (unpleasant feelings). All other withdrawals were not suspected to be related to the dose of HC. The withdrawals were evenly distributed in the two study arms and study periods.

\section{Laboratory Measurements}

Serum cortisol levels were measured by a commercially available electrochemiluminescence immunoassay (ECLIA, Roche Modular Systems) as described earlier [16].

\section{HRQoL Measures}

At the end of each treatment period, patients were asked to complete the Hospital Anxiety and Depression Scale (HADS) [17], the Multidimensional Fatigue Inventory-20 (MFI-20) [18], the RAND 36-Item Health Survey (RAND-36) [19] and the Cognitive Failures Questionnaire (CFQ) [20, 21], assessing multiple aspects of HRQoL. In addition to these questionnaires, patients were instructed to complete a daily mood and symptom diary, consisting 
of items of the Patient Health Questionnaire-15 (PHQ-15) [22], the Generalized Anxiety Disorder-7 (GAD-7) [23] questionnaire and the Patient Health Questionnaire-9 (PHQ-9) [24]. A detailed description of the questionnaires and the reference population is given in the online supplementary materials and methods.

\section{Statistics}

A power analysis performed before the study indicated that two arms of 25 patients each were required to detect an effect size of 0.4 with a two-sided alpha of 0.05 and a beta of 0.80 , even when between-test correlations are poor (0.50). An effect size of 0.4 was chosen because it was considered a relevant change with a small to moderate effect. To allow for a dropout rate of about $20 \%$, a total of 60 patients were needed.

In this study, descriptive data are presented as mean $\pm \mathrm{SD}$, median with interquartile range (IQR) in brackets, frequencies or percentages. Data on HRQoL are presented as median with IQR in brackets. Data for the PHQ-15, GAD-7 and PHQ-9 were pooled and averaged over the last 4 weeks of each treatment period to give a stable measure of the severity of symptoms during the treatment period. Normality of data was analyzed using Q-Q plots. Because of the non-normal distribution of the HRQoL data, non-parametric tests were used. In cross-over studies a treatment effect can be distinguished from a period effect as well as from a treatment-byperiod interaction effect. When a treatment effect is present, the effect can be ascribed to the dose administered. A period effect can be a result of time, for example familiarization with the study situation. A treatment-by-period interaction is often referred to as a carryover effect. To test for period effects and carryover effects, the procedure developed by Altman was used [25]. Differences in HRQoL between the lower and the higher doses were assessed with the Wilcoxon signed-rank test for paired data. Cohen's d effect sizes were calculated to give a measure of the magnitude of the difference. An effect size of $d=0.2$ was considered a small effect; $d=$ 0.5 a moderate effect and $d=0.8$ a large effect [26]. The level of significance was set at two-sided $\mathrm{p}<0.05$. All statistical analyses were performed using the Statistical Package for the Social Sciences version 22 (SPSS Inc., Armonk, N.Y., USA).

\section{Results}

\section{Study Population}

The clinical characteristics of the study population have been published previously [16]. In short, 47 patients (29 men, age $51 \pm 14$ years, range 19-73 years) completed both study periods and the data of these patients were used for further analysis. The patients' characteristics are given in online supplementary table 2 . The two groups were similar with respect to demographic and clinical characteristics, as described previously [16].

\section{Cortisol Levels}

Administration of the higher dose of $\mathrm{HC}$ resulted in significantly higher serum cortisol levels than the lower dose of $\mathrm{HC}$, both $1 \mathrm{~h}$ after ingestion of the morning dose
(925 [820-1,045] nmol/l and $620[490-730] \mathrm{nmol} / \mathrm{l}$ for the higher and lower dose, respectively, $\mathrm{p}<0.001)$ and $5 \mathrm{~h}$ after ingestion of the morning dose (305 [235-524] $\mathrm{nmol} / \mathrm{l}$ and 155 [95-270] nmol/l for the higher and lower dose, respectively, $\mathrm{p}=0.001$ ).

\section{Stress-Related Dose Adjustments}

The frequency of dose adjustments was equal during treatment with the lower dose (159 adjustments, $1.6 \%$ of the total dose administrations) and the higher dose (146 adjustments, 1.5\%). Furthermore, the number of temporary dose increments did not differ between the study groups or study periods (data not shown).

\section{Somatic Complaints, Pain and HRQoL}

On treatment with the higher dose of $\mathrm{HC}$, patients reported fewer symptoms of depression than when on treatment with the lower dose of HC ( $\mathrm{p}=0.016$ for HADS, fig. 1 , online suppl. table 3; $p=0.041$ for PHQ-9, table 1). Similarly, they reported better motivation ( $\mathrm{p}=$ $0.021)$, less general fatigue $(p=0.004)$, less mental fatigue ( $\mathrm{p}=0.003$, MFI-20, fig. 1 , online suppl. table 3 ), better physical functioning $(\mathrm{p}=0.041)$, more vitality $(\mathrm{p}=0.025)$ and better general health perception $(\mathrm{p}=$ 0.013 , RAND-36, fig. 1, online suppl. table 3). Furthermore, while receiving the higher dose of $\mathrm{HC}$, patients reported fewer somatic complaints ( $\mathrm{p}=0.023$, PHQ-15, table 1 ), particularly less pain ( $<<0.001$, fig. 2 ) and less shortness of breath $(p=0.046$, table 1$)$. In addition, while receiving the higher dose of $\mathrm{HC}$, patients reported more interest or pleasure in doing things $(\mathrm{p}=0.015)$, more energy $(p=0.007)$ and less often a disturbed eating pattern ( $\mathrm{p}=0.014$, PHQ-9, table 1$)$ compared to the lower dose. No differences in anxiety (HADS, fig. 1, online suppl. table 3; GAD-7, data not shown) and self-reported cognitive failures (CFQ, online suppl. table 3) were found between the doses. Effect sizes for the differences found ranged between 0.1 and 0.4. A significant period effect was found for depressive symptoms $(p=0.018)$, general fatigue $(\mathrm{p}=0.008)$ and mental fatigue $(\mathrm{p}=0.044)$ (online suppl. fig. 2). Graphical analysis of this effect showed that the group first receiving the lower dose of HC showed no change in reported symptoms after switching to the higher dose, whereas the group first receiving the higher dose of HC showed a significant increase in complaints after switching to the lower dose of HC. No significant carryover effects were found for the HRQoL measures, except for the blunders subscale of the CFQ $(\mathrm{p}=0.024)$. 
Fig. 1. Z-scores for HADS, MFI-20 and RAND-36 for patients treated for secondary AI $(n=47)$. Higher HADS scores indicate more anxiety and/or depression, higher MFI-20 scores indicate more fatigue, and higher RAND-36 scores indicate better QoL. Gray circles represent scores on the higher dose; black squares represent scores on the lower dose. Data represent mean \pm SEM. ${ }^{*} \mathrm{p}<0.05 .{ }^{\#}$ A significant period effect was found in addition to a significant treatment effect.

Fig. 2. Pain scores plotted for each week. For the composite pain score, the items 'stomach pain', 'back pain', 'pain in legs, arms or joints', 'headache' and 'chest pain' of the PHQ-15 were summed up, with scores ranging from 5 to 35 . Scores represent mean \pm SEM. Figures on the $\mathrm{x}$-axis are weeks.
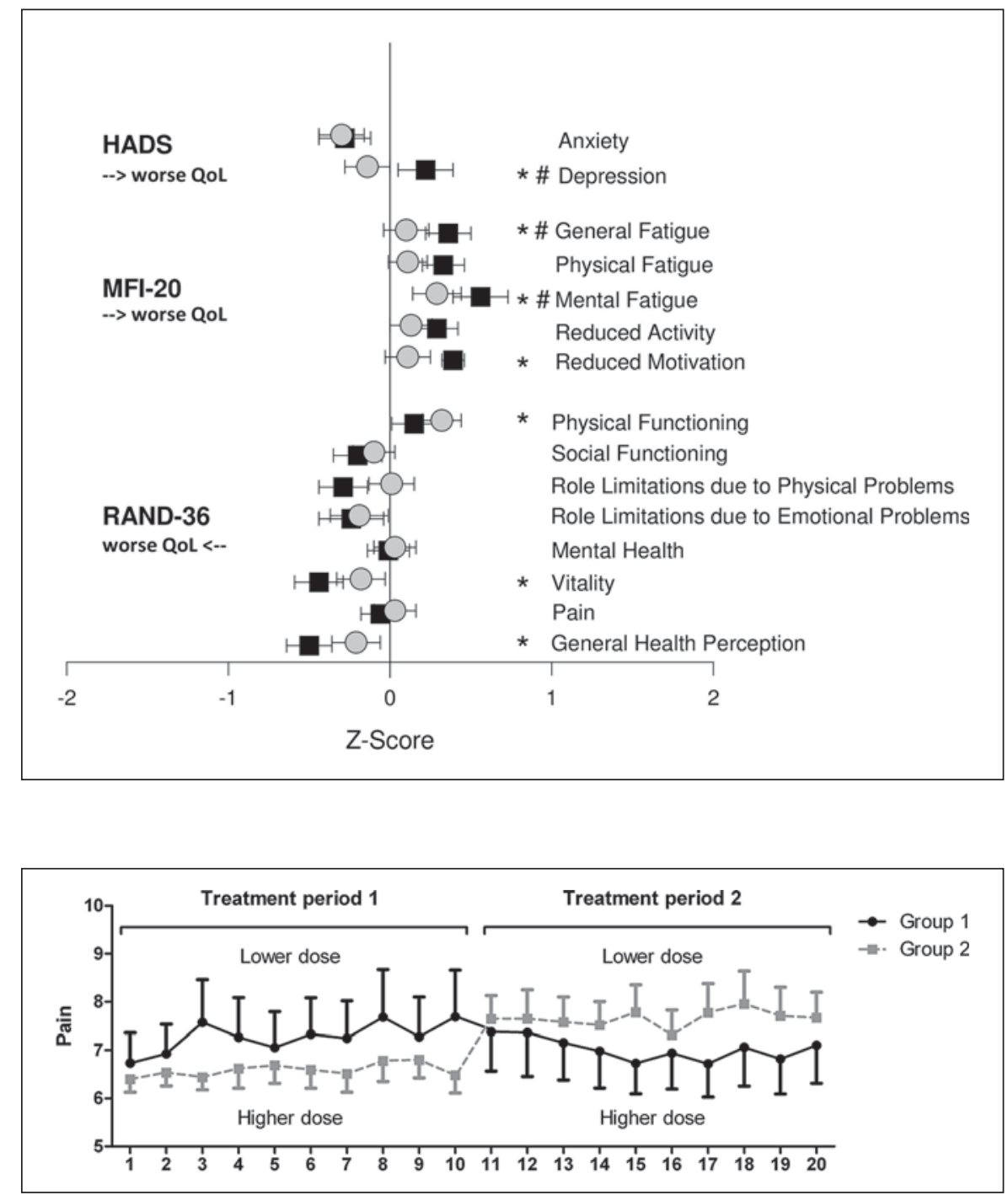

Table 1. Daily assessment of somatic complaints, anxiety and depressive symptoms in pituitary patients with secondary AI in the lowerdose and the higher-dose condition ( $\mathrm{n}=45$ due to missing data)

\begin{tabular}{|c|c|c|c|c|}
\hline & Lower-dose HC & Higher-dose HC & p value ${ }^{1}$ & Effect size \\
\hline PHQ-15 & $12.89[11.13-16.04]$ & $12.32[11.28-14.76]$ & 0.023 & 0.2 \\
\hline Back pain & $1.00[1.00-1.84]$ & $1.00[1.00-1.47]$ & 0.011 & 0.3 \\
\hline Headache & $1.04[1.00-1.80]$ & $1.04[1.00-1.54]$ & 0.037 & 0.1 \\
\hline Shortness of breath & $1.00[1.00-1.95]$ & $1.00[1.00-1.57]$ & 0.046 & 0.2 \\
\hline Feeling tired or having little energy & $1.29[1.00-2.56]$ & $1.14[1.00-2.01]$ & 0.007 & 0.2 \\
\hline Poor appetite or overeating & $1.00[1.00-1.18]$ & $1.00[1.00-1.09]$ & 0.014 & 0.2 \\
\hline
\end{tabular}

Data were pooled and averaged over the last 4 weeks of each treatment period. Higher scores indicate more complaints. The scores on the two doses are given as median [IQR]. Only significant subitems of the PHQ-15 and the PHQ- 9 are shown.

${ }^{1} \mathrm{p}$ value lower-dose $\mathrm{HC}$ vs. higher-dose $\mathrm{HC}$ by Wilcoxon signed-rank test for paired observations. 


\section{Discussion}

This randomized double-blind cross-over trial showed that patients experience benefits on various aspects of HRQoL while on a higher dose of HC substitution when compared to a lower dose of HC. These effects included, with a striking congruence, improved sense of general and mental health, improved physical functioning, fewer symptoms of depression, fewer somatic complaints, less pain and less fatigue. Our results emphasize that HRQoL is a clinically relevant aspect of HC treatment that needs to be taken into account when individualizing $\mathrm{HC}$ substitution therapy.

Overall, most of the findings appear to be related to energy and vitality. Physical health seems to be more affected than mental health. For example, with regard to depression, a close examination of the specific symptoms of the PHQ-9 showed that somatic complaints (e.g. lack of energy, disturbed eating patterns) are affected by HC dose to a greater degree than mood (e.g. feeling down, depressed or hopeless or feeling bad about oneself). Furthermore, pain and fatigue were strongly influenced by $\mathrm{HC}$ dose. On the other hand, HC dose does not seem to impact symptoms of anxiety (HADS anxiety scale, GAD-7). However, the depression subscale of the HADS assesses mostly mental aspects (e.g. 'I enjoy the things I used to enjoy', 'I can laugh and see the funny side of things'), so mental health is not unaffected. Nevertheless, the effects of HC dose on energy- and vitality-related aspects are most pronounced.

Secondary AI is often accompanied by other pituitary hormone deficiencies. Thyroid hormone deficiency, growth hormone deficiency or sex hormone deficiency could also have an effect on QoL [27]. However, in our study other pituitary hormone deficiencies were adequately replaced when necessary for at least 6 months prior to study entry and replacement therapy was held constant during the study periods. Furthermore, due to the cross-over design of the study, any remaining effect of these deficiencies would have influenced patients during both treatment periods. Therefore the effect of the other pituitary hormone deficiencies is unlikely to have influenced the results. One might argue that patients with primary AI lack these possible influential factors. However, primary AI is often accompanied by other comorbidities, e.g. thyroid disorders, gonadal disorders and diabetes, as well as treatments (e.g. fludrocortisone and dehydroepiandrosterone) which in turn have an effect on QoL.

Previous studies on HC substitution dose and HRQoL were inconclusive. In a recently published large cross-sectional study, Ragnarsson et al. [9] showed that an increas-

Hydrocortisone Treatment and Quality of Life ing dose of HC was associated with impaired HRQoL. However, due to the cross-sectional design, no conclusions could be drawn with regard to the causality of this association. In addition, previous randomized controlled trials (RCTs) showed conflicting results, mostly in contrast to our findings. A study by Alonso et al. [11] showed better general health perception on a dose of $30 \mathrm{mg} \mathrm{HC/}$ day compared to a dose of $20 \mathrm{mg} \mathrm{HC/day.} \mathrm{In} \mathrm{contrast,} \mathrm{two}$ studies comparing doses of 15,20 or $30 \mathrm{mg} \mathrm{HC/day} \mathrm{found}$ no differences in QoL $[12,13]$. However, the patient samples were small ( $\mathrm{n}=9$ for Wichers et al. [12] and $\mathrm{n}=10$ for Behan et al. [13]), which might be a reason for the lack of differences. In another RCT, a decline in physical QoL and current well-being was reported after treatment with doses of $20 \mathrm{mg} \mathrm{HC/day}$ or $5 \mathrm{mg}$ prednisone/day as compared to $15 \mathrm{mg} \mathrm{HC/day} \mathrm{[14].} \mathrm{However,} \mathrm{besides} \mathrm{the} \mathrm{dose,}$ the timing of dose administration was also altered in that study. This precludes proper interpretation of the results because not only dose but also rhythm was changed. The latter is an inherent feature of the intact hypothalamicpituitary-adrenal axis and may be responsible for the observed differences.

In our study, HRQoL was causally related to HC dose and the ensuing serum cortisol concentrations. It has previously been described that high doses of steroids may result in a state of euphoria (potentially mediated by mineralocorticoid receptors in the brain) [28]. This effect, however, was observed at supraphysiological doses of GCs. Our results extend the previous data, showing that subjective well-being is influenced at much lower doses of GCs. Although the effect sizes indicate that the observed differences were small to moderate (ranging between 0.1 and 0.4 ), they can be considered meaningful. For example, a difference of 3 points is regarded to be the minimally clinically important difference for the RAND36 [29]. We found this clinically important difference in six of the eight domains. Considering that substitution therapy is lifelong, even small contributions to perceived health are important.

One of the most striking results of our study is the effect of HC dose on pain. The relationship between cortisol and pain is not univocally reported. A study in healthy subjects showed that higher cortisol levels prior to a cold pain pressor test predicted lower subsequent pain sensitivity in men, but not in women [30]. Similarly, medically induced hypocortisolism resulted in enhanced subjective pain sensitivity [31]. On the other hand, some studies showed an inverse relationship, with higher levels being associated to greater pain sensitivity $[32,33]$ and decreases in pain thresholds [34]. Furthermore, Wingen- 
feld et al. [35] were unable to show an effect of the administration of $\mathrm{HC}$ or of dexamethasone-induced hypocortisolism on pain sensitivity. The results of the present study support the knowledge that $\mathrm{HC}$ dose plays a role in pain sensitivity and that even small changes of cortisol levels within the physiological range attenuate pain perception. This increased pain perception was not specifically located in one bodily area, suggesting a central effect of HC. In our study, bodily pain on the RAND-36 was not found to be different between the two HC doses. Although this may seem contradictory, it must be noted that the RAND36 has only two non-specific questions regarding pain, while the PHQ-15 measures pain on a daily basis based on five different bodily areas. This discrepancy is therefore likely to be a methodological issue.

Another potentially relevant finding of this study was that in the group receiving the higher dose of $\mathrm{HC}$ followed by the lower dose, a lowering of the dose resulted in a significant increase in depressive symptoms, mental fatigue and general fatigue. In comparison to the previous period, they experienced more symptoms of fatigue and depression. It might be that these patients realized 'what they were missing' once they switched to the lower dose. A decrease in reported symptoms was, however, not observed in the other group switching from the lower dose to the higher dose. Due to the design of the study, this group could not experience the 'realizing what you are missing once it is gone' effect. However, it could be expected that the same effect would be present in this group when the higher dose of $\mathrm{HC}$ was followed by a lower dose. Therefore, from a practical point of view, these findings suggest that it may be better to start with a lower dose and subsequently increase it when needed, because the reverse will possibly increase depressive symptoms, general fatigue and mental fatigue. That this period effect was not found in for example the pain scores could be due to methodological differences. Pain was assessed every day by diaries and then combined to create a total score per treatment period, while depression and fatigue were measured at the end of each treatment period only. It might be possible that these different approaches led to this discrepancy. Lower plasma cortisol levels are found in several stress-associated neuropsychiatric disorders, e.g. posttraumatic stress disorder, panic disorders and chronic pain and fatigue syndromes [36-38]. This resembles the findings in our study, with more complaints of pain and fatigue being reported by patients receiving the lower dose of $\mathrm{HC}$, an intriguing finding which points to a direct role for $\mathrm{HC}$ rather than to other hormones of the hypothalamic-pituitary-adrenal axis because feedback regula- tion is absent in our patients due to their pituitary disease. It would be of interest to study whether receptor sensitivity is of importance for those who appear particularly responsive to HC dose lowering [39].

Unfavorable effects of higher doses of GCs are known. Higher doses of GCs are associated with increased cardiovascular risk [3]. The effects of GC dose on bone mineral density remain inconclusive, with some studies reporting a linear decrease in bone mineral density with increasing HC doses in men [40], while other studies show no difference in bone mineral density between three different doses of HC [41]. Since there are no objective parameters to assess the quality of GC replacement therapy, these aspects also need to be taken into account when determining the appropriate dose for a patient. However, it should be noted that improving cardiovascular risk profile and bone strength can be achieved by a number of medical interventions, while improving HRQoL is not otherwise easily managed. Future studies should study the effects of different substitution doses on possible side effects of GC substitution therapy in RCTs.

The strength of our study is its cross-over design, making the study sufficiently powered to detect small to moderate effect sizes. In addition, we measured HRQoL not only at the end of each treatment period but also during the entire study while at the same time documenting temporary dose adjustments. Measuring HRQoL, both with diaries and retrospectively with questionnaires, resulted in a consistent pattern of complaints. Potential weaknesses include the possibility of a carryover effect inherent to the study design and the number of patients withdrawn from the study. Considering carryover effects, we found no statistical evidence for these effects, except for one subscale of self-reported cognitive functioning. Given that a wash-out period is not feasible in patients with secondary AI because they cannot be left untreated without endangering their safety, carryover effects cannot be fully excluded. However, since there was very little statistical evidence for a carryover effect, we believe this did not influence our results to any relevant extent. Accordingly, we found that several health-related aspects monitored with diaries during the treatment periods, including the effects on pain, did not change after the first week of treatment. Furthermore, even though the number of withdrawals was slightly higher than suspected, few of these withdrawals were suspected to be related to HC dose. A comparable number of study withdrawals took place in both treatment groups and periods, with the most common reason for withdrawal being protocol violations [16]. 
In conclusion, it is generally recommended to use the lowest dose of HC that relieves symptoms of GC deficiency. This advice in current guidelines remains true only when HRQoL is adequately considered in determining the appropriate dose for the individual patient. Our results show that a higher HC substitution dose will improve patient well-being.

\section{Acknowledgement}

The authors would like to thank Judith Rosmalen for her expert advice with regard to the use of daily diary questionnaires.

\section{Statement of Ethics}

The study protocol was approved by the local ethics committee of the University Medical Center Groningen, The Netherlands. Patients provided written informed consent before entering the study. This study is registered with ClinicalTrials.gov, identification number NCT01546922.

\section{Disclosure Statement}

The authors have no conflict of interest to disclose. This research did not receive any specific grant from any funding agency in the public, commercial or not-for-profit sector.

\section{References}

-1 Arlt W, Rosenthal C, Hahner S, Allolio B: Quality of glucocorticoid replacement in adrenal insufficiency: clinical assessment vs. timed serum cortisol measurements. Clin Endocrinol (Oxf) 2006;64:384-389.

2 Grossman A, Johannsson G, Quinkler M, Zelissen P: Therapy of endocrine disease: perspectives on the management of adrenal insufficiency: clinical insights from across Europe. Eur J Endocrinol 2013;169:R165R175.

- 3 Filipsson H, Monson JP, Koltowska-Haggstrom M, Mattsson A, Johannsson G: The impact of glucocorticoid replacement regimens on metabolic outcome and comorbidity in hypopituitary patients. J Clin Endocrinol Metab 2006;91:3954-3961.

-4 Mah PM, Jenkins RC, Rostami-Hodjegan A, Newell-Price J, Doane A, Ibbotson V, Tucker GT, Ross RJ: Weight-related dosing, timing and monitoring hydrocortisone replacement therapy in patients with adrenal insufficiency. Clin Endocrinol (Oxf) 2004;61:367-375.

5 Bancos I, Hahner S, Tomlinson J, Arlt W: Diagnosis and management of adrenal insufficiency. Lancet Diabetes Endocrinol 2015;3: 216-226.

6 Johannsson G, Falorni A, Skrtic S, Lennernas H, Quinkler M, Monson JP, Stewart PM: Adrenal insufficiency: review of clinical outcomes with current glucocorticoid replacement therapy. Clin Endocrinol (Oxf) 2015;82: 2-11.

7 Tiemensma J, Andela CD, Kaptein AA, Romijn JA, van der Mast RC, Biermasz NR, Pereira AM: Psychological morbidity and impaired quality of life in patients with stable treatment for primary adrenal insufficiency: cross-sectional study and review of the literature. Eur J Endocrinol 2014;171:171-182.

8 Hahner S, Loeffler M, Fassnacht M, Weismann D, Koschker AC, Quinkler M, Decker $\mathrm{O}$, Arlt W, Allolio B: Impaired subjective health status in 256 patients with adrenal insufficiency on standard therapy based on cross-sectional analysis. J Clin Endocrinol Metab 2007;92:3912-3922.

-9 Ragnarsson O, Mattsson AF, Monson JP, Filipsson Nystrom H, Akerblad AC, KoltowskaHaggstrom M, Johannsson G: The relationship between glucocorticoid replacement and quality of life in 2737 hypopituitary patients. Eur J Endocrinol 2014;171:571-579.

10 Bleicken B, Hahner S, Loeffler M, Ventz M, Decker O, Allolio B, Quinkler M: Influence of hydrocortisone dosage scheme on health-related quality of life in patients with adrenal insufficiency. Clin Endocrinol (Oxf) 2010;72: 297-304.

-11 Alonso N, Granada ML, Lucas A, Salinas I, Reverter J, Oriol A, Sanmarti A: Evaluation of two replacement regimens in primary adrenal insufficiency patients. Effect on clinical symptoms, health-related quality of life and biochemical parameters. J Endocrinol Invest 2004;27:449-454.

12 Wichers M, Springer W, Bidlingmaier F, Klingmuller D: The influence of hydrocortisone substitution on the quality of life and parameters of bone metabolism in patients with secondary hypocortisolism. Clin Endocrinol (Oxf) 1999;50:759-765.

13 Behan LA, Rogers B, Hannon MJ, O’Kelly P, Tormey W, Smith D, Thompson CJ, Agha A: Optimizing glucocorticoid replacement therapy in severely adrenocorticotropin-deficient hypopituitary male patients. Clin Endocrinol (Oxf) 2011;75:505-513.

14 Benson S, Neumann P, Unger N, Schedlowski M, Mann K, Elsenbruch S, Petersenn S: Effects of standard glucocorticoid replacement therapies on subjective well-being: a randomized, double-blind, crossover study in patients with secondary adrenal insufficiency. Eur J Endocrinol 2012;167:679-685.

15 Dullaart RP, Pasterkamp SH, Beentjes JA, Sluiter WJ: Evaluation of adrenal function in patients with hypothalamic and pituitary disorders: comparison of serum cortisol, urinary free cortisol and the human-corticotrophin releasing hormone test with the insulin tolerance test. Clin Endocrinol (Oxf) 1999;50:465471.

16 Werumeus Buning J, Brummelman P, Koerts J, Dullaart RP, van den Berg G, van der Klauw MM, Tucha O, Wolffenbuttel BH, van Beek AP: The effects of two different doses of hydrocortisone on cognition in patients with secondary adrenal insufficiency - results from a randomized controlled trial. Psychoneuroendocrinology 2015;55:36-47.

17 Zigmond AS, Snaith RP: The Hospital Anxiety and Depression Scale. Acta Psychiatr Scand 1983;67:361-370.

18 Smets EM, Garssen B, Bonke B, De Haes JC: The Multidimensional Fatigue Inventory (MFI) psychometric qualities of an instrument to assess fatigue. J Psychosom Res 1995; 39:315-325.

19 van der Zee KI, Sanderman R: The Measurement of the General Health with the RAND36, a Handbook. Groningen, Rijks Universiteit Groningen, Noordelijk Centrum voor Gezondsheidsvraagstukken, 2012.

20 Wallace JC, Kass SJ, Stanny CJ: The Cognitive Failures Questionnaire revisited: dimensions and correlates. J Gen Psychol 2002;129:238256.

21 Broadbent DE, Cooper PF, FitzGerald P, Parkes KR: The Cognitive Failures Questionnaire (CFQ) and its correlates. Br J Clin Psychol 1982;21:1-16.

22 Kroenke K, Spitzer RL, Williams JB: The PHQ-15: validity of a new measure for evaluating the severity of somatic symptoms. Psychosom Med 2002;64:258-266.

23 Spitzer RL, Kroenke K, Williams JB, Lowe B: A brief measure for assessing generalized anxiety disorder: the GAD-7. Arch Intern Med 2006;166:1092-1097.

24 Lowe B, Kroenke K, Herzog W, Grafe K: Measuring depression outcome with a brief selfreport instrument: sensitivity to change of the Patient Health Questionnaire (PHQ-9). J Affect Disord 2004;81:61-66. 
25 Altman D: Practical Statistics for Medical Research. London, Chapman and Hall, 1991.

26 Cohen J: Statistical Power Analysis for the Behavioral Sciences, ed 2. Hillsdale, Lawrence Erlbaum Associates, 1988.

27 Dekkers OM, van der Klaauw AA, Pereira AM, Biermasz NR, Honkoop PJ, Roelfsema F, Smit JW, Romijn JA: Quality of life is decreased after treatment for nonfunctioning pituitary macroadenoma. J Clin Endocrinol Metab 2006;91:3364-3369.

28 Plihal W, Krug R, Pietrowsky R, Fehm HL, Born J: Corticosteroid receptor mediated effects on mood in humans. Psychoneuroendocrinology 1996;21:515-523.

29 Hays RD, Morales LS: The RAND-36 measure of health-related quality of life. Ann Med 2001;33:350-357.

30 al'Absi M, Petersen KL, Wittmers LE: Adrenocortical and hemodynamic predictors of pain perception in men and women. Pain 2002;96:197-204.

31 Kuehl LK, Michaux GP, Richter S, Schachinger H, Anton F: Increased basal mechanical pain sensitivity but decreased perceptual wind-up in a human model of relative hypocortisolism. Pain 2010;149:539-546.
32 Goodin BR, Smith MT, Quinn NB, King CD, McGuire L: Poor sleep quality and exaggerated salivary cortisol reactivity to the cold pressor task predict greater acute pain severity in a non-clinical sample. Biol Psychol 2012;91:36-41.

33 Crettaz B, Marziniak M, Willeke P, Young P, Hellhammer D, Stumpf A, Burgmer M: Stress-induced allodynia - evidence of increased pain sensitivity in healthy humans and patients with chronic pain after experimentally induced psychosocial stress. PLoS One 2013;8:e69460.

34 Choi JC, Chung MI, Lee YD: Modulation of pain sensation by stress-related testosterone and cortisol. Anaesthesia 2012;67:1146-1151. 35 Wingenfeld K, Wolf S, Kunz M, Krieg JC, Lautenbacher S: No effects of hydrocortisone and dexamethasone on pain sensitivity in healthy individuals. Eur J Pain 2015;19:834841.

36 Yehuda R, Seckl J: Minireview: Stress-related psychiatric disorders with low cortisol levels: a metabolic hypothesis. Endocrinology 2011; 152:4496-4503.
Jezova D, Vigas M, Hlavacova N, Kukumberg $\mathrm{P}$ : Attenuated neuroendocrine response to hypoglycemic stress in patients with panic disorder. Neuroendocrinology 2010;92:112119.

38 Tak LM, Cleare AJ, Ormel J, Manoharan A, Kok IC, Wessely S, Rosmalen JG: Meta-analysis and meta-regression of hypothalamic-pituitary-adrenal axis activity in functional somatic disorders. Biol Psychol 2011;87:183194.

39 Manenschijn L, van den Akker EL, Lamberts SW, van Rossum EF: Clinical features associated with glucocorticoid receptor polymorphisms. An overview. Ann NY Acad Sci 2009; 1179:179-198.

40 Zelissen PM: Effect of glucocorticoid replacement therapy on bone mineral density in patients with Addison disease. Ann Intern Med 1994;120:207-210.

41 Ragnarsson O, Nystrom HF, Johannsson G: Glucocorticoid replacement therapy is independently associated with reduced bone mineral density in women with hypopituitarism. Clin Endocrinol (Oxf) 2012;76:246-252. 